\title{
Research on MAC Protocol for Underwater Acoustic Sensor Networks Cheng Yin ${ }^{1, a^{*}}$,Wei Wang ${ }^{1, b}$, Hui Xi ${ }^{2, c}$ \\ ${ }^{1}$ Shandong Water Conservancy Vocational College, Rizhao China \\ ${ }^{2}$ Rizhao CICC, Rizhao China

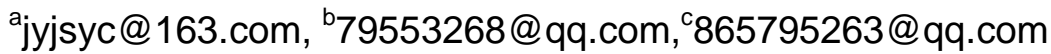

Keywords: Sensor networks; Efficiency; Underwater acoustic communication; MAC protocol.

\begin{abstract}
The underwater acoustic sensor network has been used in such wide areas as oceanographic data collection, pollution monitoring, disaster warning, offshore exploration, navigational assistant and tactical surveillance application. While the implementing of these applications lies in the key technology_- underwater acoustic communications technology.Therefore, it is a critically important mission for underwater acoustic network to design an underwater acoustic communication technology which can improve the throughput and reliability of network to the greatest extent, and at the same time, energy consumption and propagation delay can also be lowered effectively.
\end{abstract}

\section{Introduction}

An underwater acoustic sensor network is a wireless network which is built with the aid of the sound signal. It consists of a series of sensor nodes and the equipment, between nodes by underwater acoustic channel connection. We put these sensors arranged in the monitoring area, to achieve long-term underwater early warning of this area, target tracking, marine environmental factors monitoring and other tasks.

Once the sensor nodes are deployed, it is often a few months, even a year. Because the sensor nodes are powered by batteries, the energy of the battery limits the service life of the underwater sensor network to a large extent. In addition, the narrow bandwidth, multipath phenomenon and high transmission delay also reduce the efficiency of the underwater acoustic communication network. Therefore, how to design an underwater acoustic communication network structure, so that it can maximally improve the network throughput and reliability, and can reduce the energy consumption and propagation delay, for underwater acoustic sensor networks is important task.

\section{Architecture of underwater Acoustic sensor networks}

Underwater Acoustic Sensor Networks(UAN), like most other networks, are organized in a hierarchical manner. However, with the OSI protocol stack model and the TCP/IP protocol architecture, the structure of the UAN is divided into three layers, the physical layer, data link layer and network layer. The architecture of the UAN, the OSI protocol stack model and the TCP/IP protocol are compared in Figure 1. 


\begin{tabular}{|c|c|c|}
\cline { 2 - 2 } \multicolumn{1}{c|}{} & \multicolumn{1}{c}{ OSI } \\
\cline { 2 - 2 } \multicolumn{1}{c|}{ Application layer } & \multicolumn{1}{c}{ TCP/IP } \\
\cline { 2 - 2 } & Presentation layer & Application layer \\
\cline { 2 - 2 } & Session layer & Transport layer \\
\cline { 2 - 2 } & Transport layer & Network layer \\
\hline Network layer & Network layer & Network access layer \\
\hline Data link layer & Data link layer & Physical layer \\
\hline Physical layer & Physical layer & Tyen \\
\hline
\end{tabular}

Fig1. Comparison of UAN, OSI and TCP/IP Architecture

The physical layer is the basis of effective communication between nodes, which is responsible for the modulation and demodulation of data. It converts the information into a binary bit stream transmitted over the channel,then detects the signal from the noise at the receiver, and then it is reduced to the original logic signal. The data link layer is responsible for error free transmission between adjacent nodes, which is composed of LLC layer and MAC layer. The network layer is mainly used to establish the topology structure of underwater acoustic network, route control, and reasonable flow and capacity control of the line, to ensure network reliability. As a result, the design of network topology has great influence on the performance and economy of the whole network, and it is the base of realizing all kinds of network protocols ${ }^{[1,2]}$.

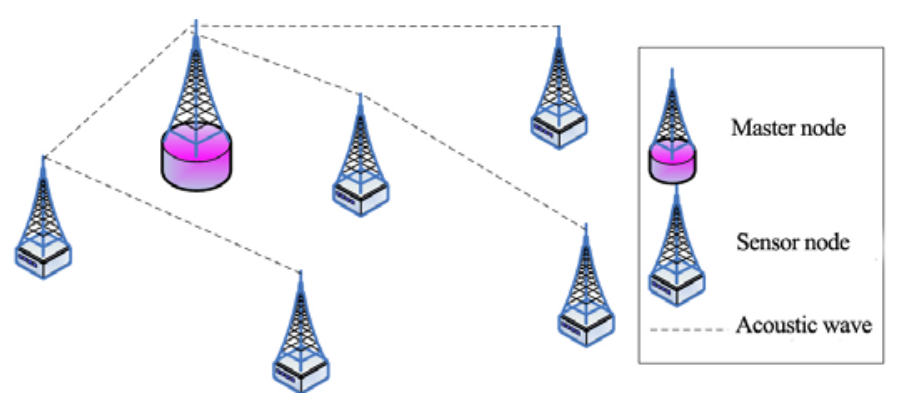

Fig2. Multi Hop Network Topology

Multi hop is a kind of multiple relay peer-to-peer topology network structure (Figure 2). The nodes in the network rely on the adjacent nodes to forward data, after several relay, finally to the main node, and then communicat with the shore based system by the master node.The coverage of the network is determined by the number of nodes, it can cover a large area, and the single node power consumption is small, so it is very suitable for the wide distribution of the sensor system in shallow water.

The MAC protocol of the data link layer ensures reliable point-to-point and point to multipoint communication, establishes a link between the nodes, and ensures that all nodes can utilize the bandwidth fairly and efficiently. In the traditional wireless network, the main evaluation indexes are throughput, bandwidth utilization, fairness and delay etc., but for the underwater acoustic communication network, the power efficiency is the first, and sometimes even to sacrifice other aspects, to obtain a higher power efficiency. This means that the traditional MAC protocol is not suitable for underwater acoustic communication networks.

In order to reduce the consumption of energy, underwater acoustic communication network protocol is usually used to "listen to sleep". When there is data to send and receive, the node will open the communication module to send or listen. If there is no data to send and receive, the node will control the communication module to enter the sleep state, thereby reducing energy consumption. In order to make the node in sleep do not miss the data, or reduce the excessive listenin, the neighbor nodes need to coordinate between listening and sleep cycle. 


\section{Slotted FAMA ${ }^{[3]} 、$ ALOHA-CS $S^{[4]}$ and TDMA ${ }^{[5]}$ protocol}

We select the existing MAC protocols which are representative: Slotted FAMA, ALOHA-CSand TDMA, Compare its performance through simulation experiments.

The Slotted FAMA protocol divides the time into small time slot $\mathrm{T}$, and the length of the $\mathrm{T}$ should ensure that the nodes in the communication range are able to receive. The protocol also uses the RTS-CTS-DATA-ACK mechanism, RTS/CTS/DATA/ACK can only be used in the time slice of the start of transmission. Among them, the control package takes up a time slice, and the data packet can last a plurality of time slices.

In Aloha-CS protocol, If the node listens to the channel on which data is sent, it does not send data., otherwise the data can be sent directly.

In the TDMA protocol, time is divided into several time slots, and the nodes can transmit data in their own time slots.

MAC frame in Slotted FAMA is divided into four types,RTS,CTS,DATA and ACK. Among them, RTS,CTSand ACK is the control frame, DATA is data frame. Aloha-CS and TDMAOnly have data frame.

The control frame consists of frame type, source node ID ,destination node ID and cyclic redundancy check(CRC), as shown in Figure 3.

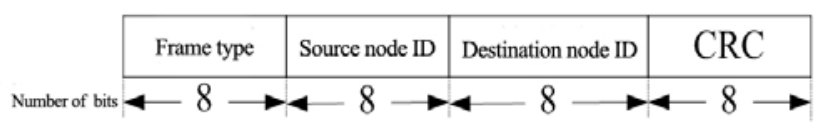

Fig 3.Structure Of the Control Frame

Among them, the frame type occupies 2 bits. 00 means RTS frames, 01 means CTS frames, 10 means DATA frames, 11 means ACK frames.Counter means the data frame numberto be transmitted.

The data frameconsists of frame type, source node ID ,destination node ID,dataand cyclic redundancy check, as shown in Figure 4.The length of data depends on the situation.

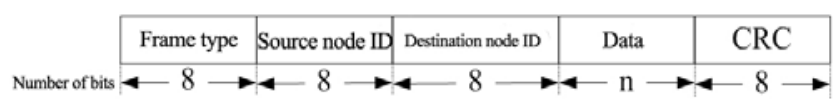

Fig 4.Structure Of the Data Frame

Figure 5 describes the underwater acoustic channel propagation model. From the transmitter (marked in the figure T) issued by the sound through the 5 different ways to reach the receiver (marked as $\mathrm{R}$ in the figure) ${ }^{[6]}$.

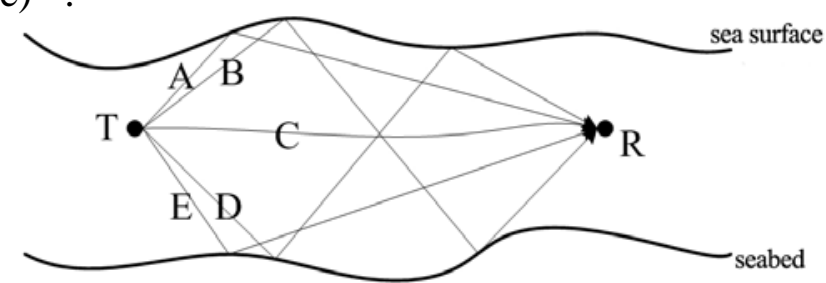

Fig 5. Acoustic Propagation Model for Underwater Acoustic Channel

1) The sound wave emitted by the transmitter is reflected by the sea surface to the receiver, which is expressed as A;

2) The sound wave emitted by the transmitter is first reflected by the sea surface, and then through the seabed to reach the receiver, the symbol is $\mathrm{B}$;

3 ) the direct sound wave, the mark is $C$;

4) The sound wave emitted by the transmitter is first reflected by the seabed, and then the though the sea surface reaches the receiver. The symbol is D;

5) the signal emitted by the transmitter reaches the receiver through the sea surface, which is marked as E.

In this paper, the underwater sensor network is a multi hop and shallow sea network. Asynchronous half duplex mode was adopted for the communication between nodes in the network, 
channel band $10 \mathrm{kHz}$, the control frame length 32bits, data frame length 512 bits (and therefore according to the definition of the data frame structure, the length of the data portion of the frame is 480bit), the transmission rate for 256bit/s. The network consists of 16 nodes, nodes are deployed in $4 \mathrm{~km} * 4 \mathrm{~km}$ in the region, one of the sink nodes, multi hop network topology structure. The bit error rate of the underwater acoustic channel is fixed value, $10-5$ bit rate, and the transmission rate of the underwater acoustic signal is $1500 \mathrm{~m} / \mathrm{s}$. Fig 6.is network deployment diagram for simulation experiment.

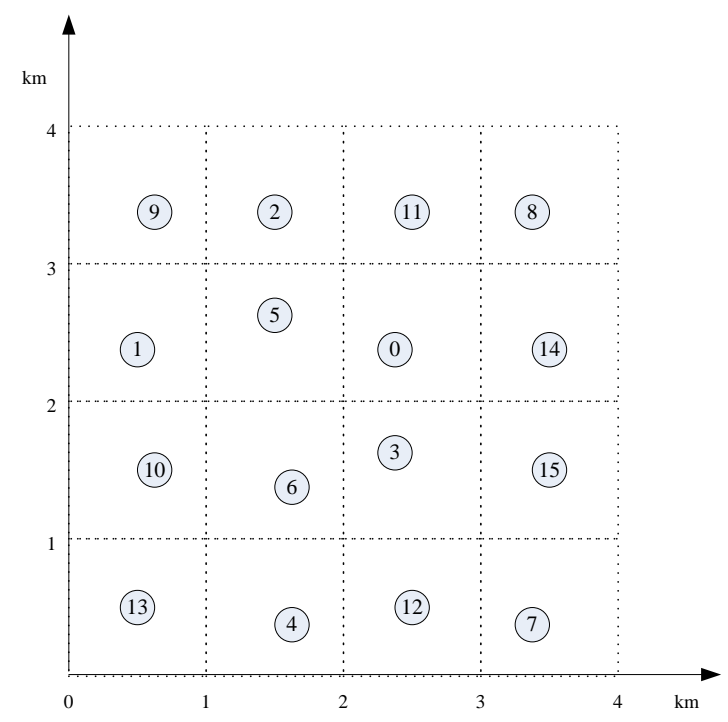

Fig 6. Network Deployment Diagram

Frame is usually used to represent sending a standard length of time. In this simulation, the frame is $2 \mathrm{~s}$.

Figure 7 describes the change in the average throughput of the system, which is a function of the network load (the total number of data frames sent per unit time). From the above, we can see that the slotted FAMA protocol requires a large number of control packets (75\% of the packet is control packets), and need a long time slice, resulting in lower performance of the protocol; The performance of ALOHA-CS is higher when the network load is low, the maximum is 0.135 , but with the increase of load, the probability of packet collision increases, so the performance of the protocol is also decreased; TDMA protocol assigns a fixed time slice for each node, it can guarantee the package in the network does not produce conflict. Therefore, the performance is better when the network load is higher, and the performance is basically maintained at 0.176 when the network load is greater than 2 .

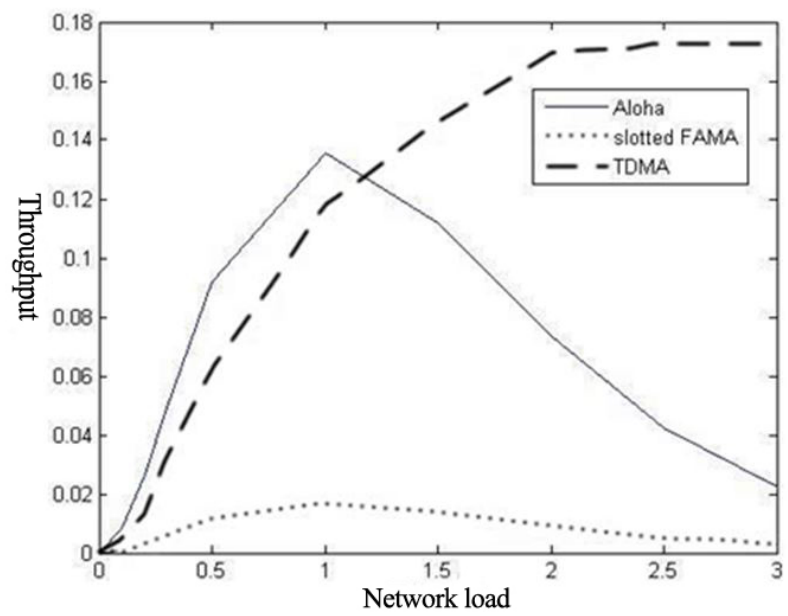

Fig 7.Performance Comparison of Slotted FAMA、ALOHA-CS， TDMA (matlab) 


\section{A Hybrid MAC protocol}

Through the analysis of the above protocols, we find that the ALOHA-CS protocol has better performance when the network load is low. The TDMA protocol is suitable for higher network load. In view of the above situation, we proposed a hybrid MAC protocol. When the network load is low, the ALOHA-CS protocol is adopted, and the load reaches a critical point (K), and then the TDMA protocol is adopted.

Each node maintains a load parameter $(\mathrm{k})$, according to data packets generated by themselves and data table from the neighbor node to determine the load parameters. Node has a packet to be sent, the first comparison with $\mathrm{K}$ and $\mathrm{k}$. If $\mathrm{k} \leqq \mathrm{K}$, first Interception Channel. If the channel data is being transmitted, then retreat for a period of time to start sending; If $\mathrm{k}>\mathrm{K}$, you first determine whether it is their own time slice, if it is to send data directly, otherwise wait until their time of arrival and then begin to send data. Figure 8 describes the flow chart of data transmission in this protocol.

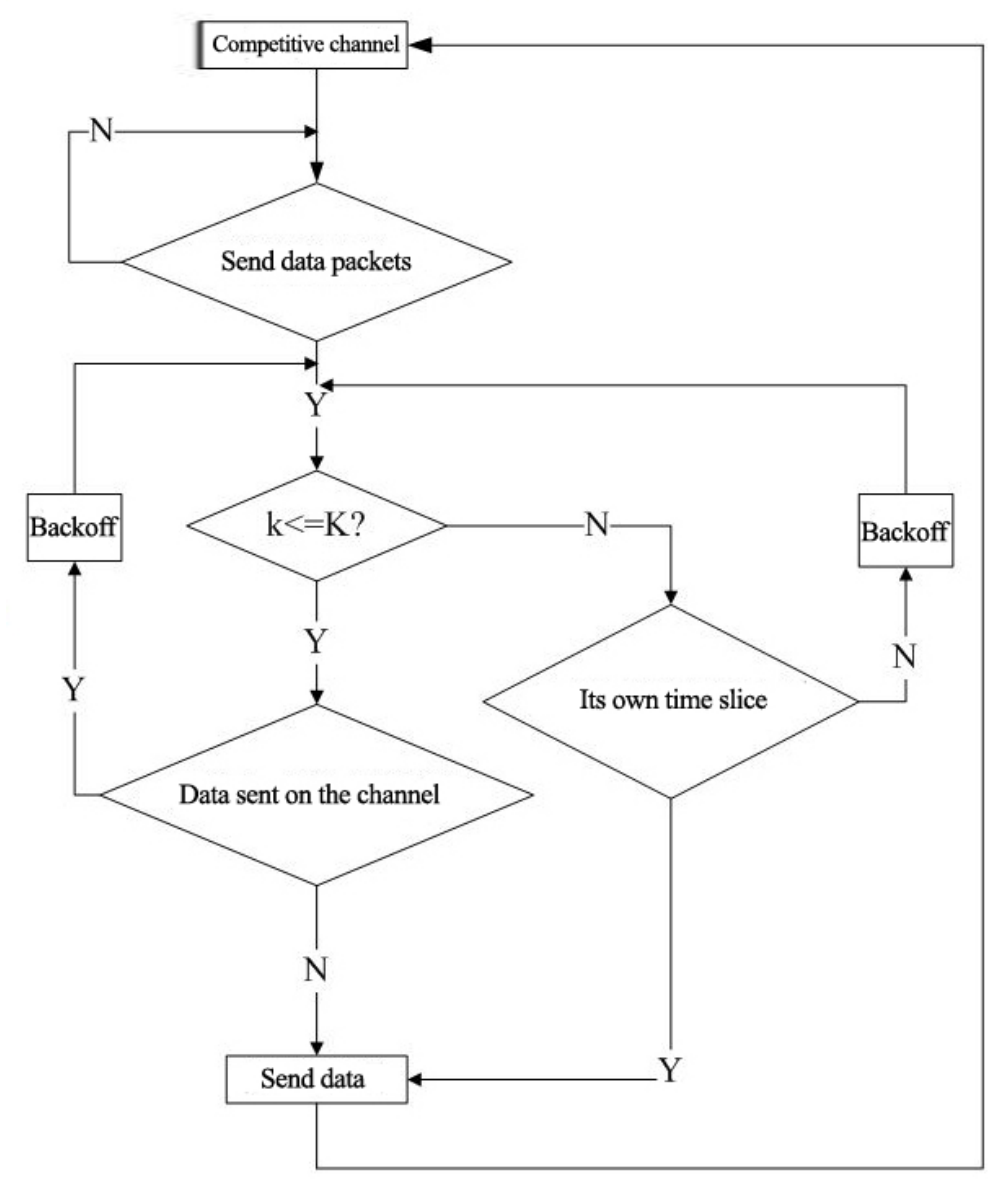

Fig 8. Data Transmission Flow Chart of Hybrid MAC Protocol

\section{Conclusion}

We simulated the design of the protocol. The network consists of 16 nodes, one of the sink nodes, channel band $10 \mathrm{kHz}$, data frame length 512 bits,the transmission rate for 256bit/s. The nodes are deployed in the area of $4 \mathrm{~km} * 4 \mathrm{~km}$, multi hop network topology structure. The bit error rate of the underwater acoustic channel is fixed value, 10-5 bit rate, and the transmission rate of the underwater acoustic signal is $1500 \mathrm{~m} / \mathrm{s}$. Figure 9 describes the variation of the throughput of the hybrid MAC protocol. The acoustic sensor can be used in different occasions, the size of network, topology, data transmission rate and other factors are not the same. Therefore, the value of $\mathrm{K}$ is not fixed (in this simulation, we take $\mathrm{K}=1.2$ ), according to the specific circumstances of the network to get. 


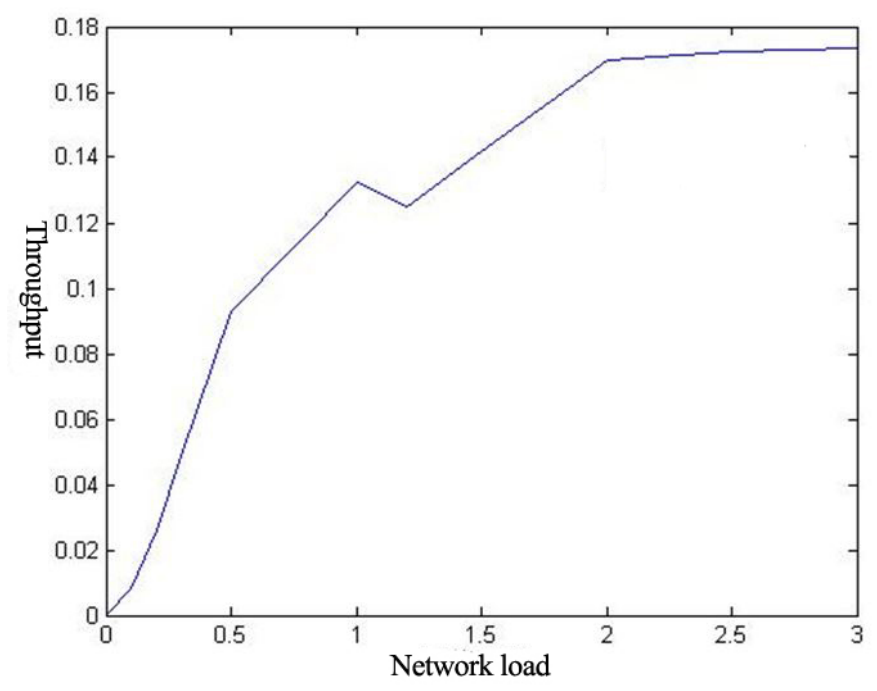

Fig 9. Performance of Hybrid MAC Protocol

\section{References}

[1] Ethem M Sozer, MilicaStojanovic, John G Proakis. Underwater acoustic networks[J]. IEEE J Oceanic Eng, 2000,25(1):72-83.

[2] John G Proakis, Joseph A Rice, MilicaStojanovic. Shallowwater acoustic networks[J]. IEEE Communication Magazine, 2001:114-119.

[3] M. Molins and M. Stojanovic. "Slotted FAMA: a MAC Protocol for Underwater Acoustic Networks” [R]. In Proc. of MTS/IEEE OCEANS. Boston, MA, USA, Sep. 2006.

[4] N.Chirdchoo, W-S.Soh, and K.C.Chua, "Aloha-based MAC Protocols with Collision Avoidance for Underwater Acoustic Networks,” [R] in INFOCOM 2007, Anchorage, Alaska, USA, May 2007, pp. 2271-2275.

[5] Hong Lu, GuoZhong-wen,A TDMA-based MAC Protocol in Underwater Sensor Networks, $[R]$ the 4th international conference on wireless communications, networking and mobile computing.

[6] Bo Sun, EnCheng, XiaoliOu, Research and Simulation of shallow water acoustic channel, Wireless Communication Technology [J], Third.2006 